\section{Apolipoprotein B: novel indicator of elevated intraocular pressure}

JH Son ${ }^{1}$, YK Chung ${ }^{2}$ and JS Son ${ }^{3}$

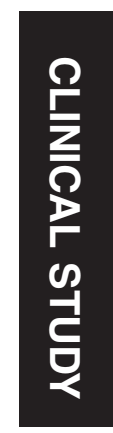

\begin{abstract}
Purpose Many studies have reported associations between elevated intraocular pressure (IOP) and systemic health parameters, which suggest a common mechanism links IOP elevation and various related cardiometabolic risk factors.

Furthermore, according to a recent study, serum apolipoprotein B (APO B) level is a predictor of coronary artery disease. This study was undertaken to analyse the relationship between serum apolipoprotein levels and IOP.

Methods Healthy people (28 852) who attended a community hospital for a health checkup between January 2011 and December 2013 were enroled in the study. We measured age, body mass index (BMI), systolic blood pressure (SBP), diastolic blood pressure (DBP), serum levels of total cholesterol (TC), triglyceride (TG), high-density lipoprotein cholesterol (HDL-C), low-density lipoprotein cholesterol (LDL-C), and apolipoprotein A1
\end{abstract} (APO A1) and APO B, APO B/APO A1 ratios, and IOP.

Results Univariate regression analysis showed IOP was positively correlated with BMI, SBP, DBP, TC, LDL-C, TG, APO B, and APO B/APO A1 $(P<0.001)$, and negatively correlated with HDL-C $(P<0.001)$. On the other hand, multivariate regression analysis adjusted for age, BMI, SBP, and DBP, revealed IOP was positive correlated with TC, TG, LDL-C, APO B, and APO B/APO A1, and negatively correlated with HDL-C (all <0.05).

Conclusions Among the various lipid profiles investigated, APO B was found to be most strongly correlated with IOP, regardless of sex. Additional studies are required to confirm the validity of apolipoprotein level as an index for predicting IOP.

Eye (2015) 29, 1315-1320; doi:10.1038/eye.2015.37;

published online 20 March 2015

\section{Introduction}

Glaucoma is a progressive ophthalmological disease characterised by optic disc cupping and visual field defects that can result in loss of vision. ${ }^{1}$ Some previous studies have concluded that family histories of glaucoma, severe myopia, or high IOP constitute major risk factors. ${ }^{2}$ Of these identified risk factors, IOP has received attention for many years as a potential modifiable risk factor. ${ }^{3-5}$

Although the mechanism underlying IOP elevation are not fully understood, a recent study reported associations between systemic health parameters, such as, hypertension, diabetes, and obesity indexes (eg, waist circumference and BMI) and IOP elevation. ${ }^{6-9}$ Hypertension, diabetes, dyslipidemia, and obesity were also found to be associated with cardiometabolic risk factors, which suggests that a common underlying mechanism links IOP elevation and related cardiometabolic risk factors. Furthermore, many previous studies have demonstrated relationships between IOP and cardiometabolic risk factors. ${ }^{10,11}$

Dyslipidemia is a well-known risk factor of coronary artery diseases and IOP elevation, ${ }^{12-15}$ and of the known risk factors of coronary artery disease, Apo B was reported recently to be the most useful indicator of coronary artery disease. ${ }^{16}$

However, relatively few studies have investigated the relationship between apolipoprotein level and IOP. Therefore, in this study, we analysed relationships between IOP and lipid profiles, such as, APO A1 and APO B levels, and APO $\mathrm{B} / \mathrm{APO} \mathrm{A} 1$ ratio.

\section{Subjects and methods}

\section{Study subjects}

Healthy examinees (29 762) who attended a health promotion centre at Samsung Changwon Hospital, Sungkyunkwan University, South
${ }^{1}$ Department of Ophthalmology, Yeungnam University College of Medicine, Daegu, South Korea

${ }^{2}$ Department of Occupational and Environmental Health, Hallym Sacred Heart Hospital, Hallym Medical Korea

${ }^{3}$ Department of Occupational and Environmental Medicine, Samsung Changwon Hospital, Sungkyunkwan University School of Medicine, Changwon, South Korea

Correspondence: JS Son, Department of Occupational and Samsung Changwon Hospital, Sungkyunkwan University School of Medicine, Changwon, 158, Palyong-ro, MasanHoiwongu, Changwon-si, Gyeongsangnam-do 630-723, South Korea Tel: +82 552906274 ; Fax: +82 552906472 .

E-mail: realcatson@ naver.com

or YK Chung, Department of Occupational And Environmental Medicine, Hallym University Sacred Heart Hospital, Anyang city, South Korea

Tel: +82 31380 1592;

Fax: +82 313801583.

E-mail: yk.chungmd@ gmail.com

Received: 29 August 2014 Accepted in revised form: 4 February 2015 Published online: 20 March 2015 University, Anyang, South Environmental Medicine, 
Korea, between January 2011 and December 2013 were initially considered as study subjects.

Before testing, each participant was interviewed by a trained nurse about previous health problems and medical history, including ocular diseases. A written informed consent was obtained from all participants and participants with a history of intraocular disease or surgery (in at least in one eye), those receiving medical treatment for glaucoma, and those who did not agree to involvement in this study were excluded. Finally, 28852 healthy examinees were included in the present study.

Before implementation, the study protocol was reviewed and approved by the institutional review board of our institution. All tests were performed after obtaining participant consent.

\section{Anthropometric and blood pressure measurements}

Heights and weights were measured using an automatic height-weight measuring instrument (GL-150, G-TECH International, Seoul, South Korea); subjects removed shoes and wore light clothes. BMI was calculated by dividing body weight by height squared $\left(\mathrm{kg} / \mathrm{m}^{2}\right)$. Blood pressure was recorded in a comfortable sitting position and using an automatic blood pressure gauge (Jawon Medical, Seoul South Korea) in the morning (between 0800 and 1000 hours) after a 10-min rest period. To ensure accuracies, subjects were requested not to drink coffee or smoke within $1 \mathrm{~h}$ of BP testing. Systolic and diastolic blood pressures were measured twice and results were averaged.

\section{Ophthalmologic examination}

All subjects underwent an ophthalmological examination, which included best-corrected visual acuity based on the Snellen chart and IOP measurements by noncontact tonometry (Topcon CT60, Topcon, Tokyo, Japan). IOP was measured on the centre of the cornea three times, and mean values were calculated for each eye. To avoid interexaminer variance, IOP measurements were performed by a trained nurse. All ophthalmologic examinations were performed between 0800 and 1000 hours. The averages of both eyes were used in the analysis.

\section{Lipid profile examination}

Blood samples were obtained after fasting for $8 \mathrm{~h}$ and centrifuged at $3500 \mathrm{rpm}$ for $10 \mathrm{~min}$ to obtain serum, which was analysed for TC, TG, HDL-C, and LDL-C using enzymatic methods (Histachi Modular DPP, Roche, Tokyo, Japan).

Plasma APO A1 and APO B concentrations were measured using immune turbidimetric immunoassay (Histachi Modular P800, Roche, Tokyo, Japan).

\section{Statistical analysis}

To analyse the baseline characteristics of study participants, we calculated mean $\pm \mathrm{SD}$; differences between men and women were determined using the $t$-test.

SBP, DBP, TC, TG, LDL-C, HDL-C, APO A1, and APO $\mathrm{B}$ data were log-transformed because of the skewed natures of their distributions. Univariate linear regression was used to investigate the association between IOP and lipid profiles, and multivariate linear regression was used to analyse relations between lipid profiles and IOP after adjusting for confounding variables.

The analyses was performed using SPSS version 18.0 for Windows (SPSS Inc., Chicago, IL, USA), and P-values of $<0.05$ were considered statistically significant.

\section{Results}

\section{Characteristics of the study participants}

Table 1 summarizes the characteristics of the 28852 healthy examinees (16 784 men and 12068 women). Mean subject age was $41.66 \pm 7.47$ years (range $19-74$ years). No significant difference was observed between the ages of men and women. However, BMI, TC, TG, LDL-C, SBP, DBP, APO B, APO B/APO A1, and both IOPs were significantly higher in men, and HDL-C and APO A1 levels were significantly higher in women.

\section{Associations between IOP and lipid profiles}

Univariate regression analysis of the relationships between IOP and BMI, SBP, DBP, TC, LDL-C, TG, APO B, and APO B/APO A1 showed all were positively and significantly correlated $(P<0.001$; Table 2$)$. HDL-C showed a negative correlation $(P<0.001)$ with IOP, whereas APO A1 showed a nonsignificant negative correlation $(P=0.379)$. IOP also increased with age, but not significantly so $(P=0.063)$.

Regarding gender differences, a male gender was negatively correlated with age and HDL-C $(P<0.001)$ but positively correlated with BMI, SBP, DBP, TC, LDL-C, APO $\mathrm{B}$, and APO B/APO A1 ratio $(P<0.001)$. However, the between age and APO A1 was not significant $(P=0.663)$. A female gender was negatively correlated with HDL-C $(P<0.001)$ but nonsignificantly with APO A1 $(P=0.624)$. The remaining variables were similar for men and women.

\section{Multivariate-adjusted linear regression analysis}

Multivariate regression analysis adjusted for IOP-related factors, that is, age, BMI, SBP, and DBP revealed significant positive correlations between IOP and TC, TG, LDL-C, APO A1, APO B, and APO B/APO A1, and a significant negative correlation with HDL-C (Table 3). 
Table 1 Clinical characteristics of the study participants

\begin{tabular}{lcrrr}
\hline Variable & Total $(\mathrm{n}=28$ 852) & Men $(\mathrm{n}=16784)$ & Women $(\mathrm{n}=12$ 068) & P-value \\
\hline Age (years) & $41.66 \pm 7.47$ & $41.79 \pm 7.70$ & $41.49 \pm 7.11$ & 0.101 \\
BMI (kg/m $\left.{ }^{2}\right)$ & $23.79 \pm 3.12$ & $24.39 \pm 2.89$ & $22.96 \pm 3.26$ & $<0.001$ \\
SBP (mm Hg) & $123.02 \pm 16.32$ & $125.09 \pm 15.57$ & $120.17 \pm 16.90$ & $<0.001$ \\
DBP (mm Hg) & $73.37 \pm 11.47$ & $74.06 \pm 11.30$ & $72.40 \pm 11.63$ & $<0.001$ \\
TC (mg/dl) & $197.52 \pm 35.16$ & $200.74 \pm 34.74$ & $193.06 \pm 36.27$ & $<0.001$ \\
TG (mg/dl) & $111.48 \pm 80.70$ & $126.51 \pm 87.31$ & $90.60 \pm 64.94$ & $<0.001$ \\
LDL-C (mg/dl) & $126.15 \pm 32.71$ & $131.05 \pm 32.50$ & $119.37 \pm 31.80$ & $<0.001$ \\
HDL-C (mg/dl) & $58.94 \pm 14.54$ & $55.34 \pm 13.36$ & $63.97 \pm 14.65$ & $<0.001$ \\
Apo A1 (mg/dl) & $147.41 \pm 25.96$ & $143.56 \pm 24.71$ & $152.76 \pm 26.72$ & $<0.001$ \\
Apo B (mg/dl) & $95.95 \pm 24.39$ & $101.21 \pm 23.95$ & $88.63 \pm 23.09$ & $<0.001$ \\
Apo B/Apo A-1 & $0.68 \pm 0.23$ & $0.72 \pm 0.22$ & $0.61 \pm 0.20$ & $<0.001$ \\
Rt. IOP (mm Hg) & $14.94 \pm 2.69$ & $15.34 \pm 2.73$ & $14.39 \pm 2.55$ & $<0.001$ \\
Lt. IOP (mm Hg) & $14.96 \pm 2.69$ & $15.34 \pm 2.73$ & $14.43 \pm 2.55$ & $<0.001$ \\
Average IOP (mm Hg) & $14.95 \pm 2.58$ & $15.34 \pm 2.60$ & $14.41 \pm 2.44$ & $<0.001$ \\
\hline
\end{tabular}

Abbreviations: APO A1, apolipoprotein A1; APO B, apolipoprotein B; BMI, body mass index; DBP, diastolic blood pressure; HDL-C, high-density lipoprotein cholesterol; IOP, intraocular pressure; LDL-C, low-density lipoprotein cholesterol; SBP, systolic blood pressure; TC, total cholesterol; TG, triglyceride.

The data are presented as mean \pm SD values. ${ }^{a}$ Independent $t$-test: between the men and women.

Table 2 Univariate linear regression analysis for associations with intraocular pressure

\begin{tabular}{|c|c|c|c|c|c|c|}
\hline \multirow{2}{*}{ Variable } & \multicolumn{2}{|c|}{ Total $(\mathrm{n}=28$ 852) } & \multicolumn{2}{|c|}{ Male $(\mathrm{n}=16784)$} & \multicolumn{2}{|c|}{ Female $(\mathrm{n}=12068)$} \\
\hline & $\beta \pm S E$ & P-value ${ }^{\mathrm{a}}$ & $\beta \pm S E$ & P-value ${ }^{\mathrm{a}}$ & $\beta \pm S E$ & P-value \\
\hline Age & $0.004 \pm 0.002$ & 0.063 & $-0.018 \pm 0.003$ & $<0.001$ & $0.030 \pm 0.003$ & $<0.001$ \\
\hline BMI & $0.148 \pm 0.005$ & $<0.001$ & $0.139 \pm 0.007$ & $<0.001$ & $0.100 \pm 0.007$ & $<0.001$ \\
\hline $\mathrm{SBP}^{\mathrm{b}}$ & $10.649 \pm 0.005$ & $<0.001$ & $9.710 \pm 0.369$ & $<0.001$ & $9.469 \pm 0.368$ & $<0.001$ \\
\hline $\mathrm{DBP}^{\mathrm{b}}$ & $7.822 \pm 0.222$ & $<0.001$ & $6.735 \pm 0.301$ & $<0.001$ & $8.117 \pm 0.319$ & $<0.001$ \\
\hline $\mathrm{TC}^{\mathrm{b}}$ & $3.190 \pm 0.197$ & $<0.001$ & $2.775 \pm 0.265$ & $<0.001$ & $2.236 \pm 0.287$ & $<0.001$ \\
\hline $\mathrm{TG}^{\mathrm{b}}$ & $1.986 \pm 0.063$ & $<0.001$ & $1.710 \pm 0.083$ & $<0.001$ & $1.304 \pm 0.106$ & $<0.001$ \\
\hline LDL-C $^{\mathrm{b}}$ & $1.990 \pm 0.129$ & $<0.001$ & $1.077 \pm 0.178$ & $<0.001$ & $1.672 \pm 0.188$ & $<0.001$ \\
\hline HDL-C $^{b}$ & $-2.555 \pm 0.142$ & $<0.001$ & $-1.263 \pm 0.195$ & $<0.001$ & $-1.615 \pm 0.221$ & $<0.001$ \\
\hline $\mathrm{APO} A 1^{\mathrm{b}}$ & $-0.087 \pm 0.201$ & 0.379 & $1.844 \pm 0.271$ & 0.663 & $-0.145 \pm 0.296$ & 0.624 \\
\hline $\mathrm{APO} \mathrm{B}^{\mathrm{b}}$ & $3.381 \pm 0.133$ & $<0.001$ & $2.621 \pm 0.189$ & $<0.001$ & $2.320 \pm 0.197$ & $<0.001$ \\
\hline APO B/APO A1 & $1.390 \pm 0.069$ & $<0.001$ & $0.688 \pm 0.092$ & $<0.001$ & $1.133 \pm 0.114$ & $<0.001$ \\
\hline
\end{tabular}

Abbreviations: APO A1, apolipoprotein A1; APO B, apolipoprotein B; BMI, body mass index; DBP, diastolic blood pressure; HDL-C, high-density lipoprotein cholesterol; IOP, intraocular pressure; LDL-C, low-density lipoprotein cholesterol; SBP, systolic blood pressure; SE, standard errors; TC, total cholesterol; TG, triglyceride. ${ }^{a}$ Univariate linear regression analysis. ${ }^{\mathrm{b}}$ Log-transformed values were used for the analysis.

Table 3 Multivariable-adjusted linear regression analysis for associations with intraocular pressure

\begin{tabular}{lccc}
\hline Variable & Total $^{\mathrm{a}} \beta \pm S E(P)^{\mathrm{b}}$ & Male $^{\mathrm{a}} \beta \pm S E(P)^{\mathrm{b}}$ & Female $^{\mathrm{a}} \beta \pm S E(P)^{\mathrm{b}}$ \\
\hline TC $^{\mathrm{c}}$ & $1.535 \pm 0.098(<0.001)$ & $1.540 \pm 0.265(<0.001)$ & $0.621 \pm 0.293(0.034)$ \\
LDL-C $^{\mathrm{c}}$ & $0.887 \pm 0.131(<0.001)$ & $0.393 \pm 0.177(0.026)$ & $0.569 \pm 0.195(0.003)$ \\
TG $^{c}$ & $1.195 \pm 0.068(<0.001)$ & $1.067 \pm 0.089(<0.001)$ & $0.613 \pm 0.111(<0.001)$ \\
HDL-C $^{\mathrm{c}}$ & $-1.273 \pm 0.147(<0.001)$ & $-0.313 \pm 0.202(0.021)$ & $-0.803 \pm 0.223(<0.001)$ \\
Apo A-1 & $0.512 \pm 0.198(0.010)$ & $1.913 \pm 0.270(<0.001)$ & $0.175 \pm 0.290(0.546)$ \\
Apo B & $1.695 \pm 0.140(<0.001)$ & $1.544 \pm 0.194(<0.001)$ & $1.038 \pm 0.209(<0.001)$ \\
Apo B/Apo A-1 & $0.707 \pm 0.073(<0.001)$ & $0.262 \pm 0.095(0.006)$ & $0.433 \pm 0.120(<0.001)$ \\
\hline
\end{tabular}

Abbreviations: APO A1, apolipoprotein A1; APO B, apolipoprotein B; BMI, body mass index; DBP, diastolic blood pressure; HDL-C, high-density lipoprotein cholesterol; IOP, intraocular pressure; LDL-C, low-density lipoprotein cholesterol; SBP, systolic blood pressure; SE, standard errors; TC, total cholesterol; TG, triglyceride. ${ }^{a}$ Adjusted for age, BMI, SBP, and DBP. ${ }^{b}$ Multiple linear regression analysis. ${ }^{c}$ Log-transformed values were used for the analysis.

IOP was a positive correlation with APO A1 at the same level of significance in males $(P<0.001)$ as when including both sexes, whereas a negative nonsignificant correlation was observed in females $(P=0.546)$. However, for
HDL-C, which is related to APO A1, a significantly negative correlation was observed with IOP for both sexes. Of the various lipid indices, APO B had the highest correlation with IOP, regardless of sex. 


\section{Discussion}

The results of the univariate regression analysis conducted indicated that IOP is significantly and positively correlated with TC, TG, LDL-C, APO B, and APO B/APO A1 but significantly and negatively correlated with HDL-C.

Reports indicate that IOP increases with age among Caucasians. ${ }^{6-8}$ In contrast, in Japanese studies $9,17,18$ and a Korea study ${ }^{19}$ IOP was found to decrease with age. This difference has been attributed to racial and hormonal influences and environmental differences. ${ }^{19}$ However, in the other study, IOP tended to increase with age, which we attribute to Westernization of the Asian diet, especially, among younger adults, which exhibit higher rates of hypertension and obesity and higher IOP.9,15 On the other hand, older males did show a significant decrease in IOP in this study, whereas females showed a significantly increase in IOP with age.

A recent study reported that South East Asian men showed a significantly negative correlation between age and IOP, whereas women showed an insignificant negative correlation. ${ }^{20}$ Such gender-associated differences may be due to hormonal differences or indicate a transition to a Western pattern. Additional studies are needed to determine whether the previous studies on Asian populations that showed a negative correlation between age and IOP reflect a change caused by Westernization or whether they are the result of genetic or racial characteristics.

Many studies have indicated that obese individuals have more orbital fat and greater episcleral pressure, which reduces aqueous humour outflow and increases IOP. 9,21,22 Obesity is also a risk factor of hypertension, and high blood pressure has been reported to increase pressure in the intraocular ciliary artery, and thereby, to stimulate the generation of aqueous humour, which increases IOP. It is has been well established that blood pressure is correlated with IOP, 22,23 and similarly, in the present study, BMI and blood pressure were both positively correlated with IOP.

IOP is known to increase with serum TC level. ${ }^{15}$ Klein et al ${ }^{24}$ suggested more orbital adipose tissue is produced at higher serum TC levels, and that this increases orbital pressure, which in turn induces an increase in episcleral pressure and a decrease in aqueous humour outflow, thus increasing IOP.

Higher fat consumption increases the risk of obesity, which increases TG levels and decreases HDL-C levels. Furthermore, sclerotic changes in arteries are induced and serum osmolality is increased, which increase episcleral pressure, and thus, increasing IOP. ${ }^{17}$

Unlike many reports that indicated IOP to be positively correlated with serum TC and TG levels, no consistent results indicate the correlation of IOP with HDL-C and
LDL-C. Although a report indicated a positive correlation between HDL-C and IOP, ${ }^{23}$ another report conversely indicated a negative correlation. ${ }^{15,25}$

In this study, univariate regression showed a negative correlation between HDL-C and IOP but a positive correlation between LDL-C and IOP, which are consistent with the findings of a recent study. ${ }^{26}$ Even though multivariate regression analysis adjusted for age, BMI, SBP, and DBP showed a negative correlation between HDL-C and IOP, a positive correlation between LDL-C and IOP, previous studies differ markedly regarding the natures of the correlations between IOP and HDL-C or LDL-C, and thus, additional studies are needed to clarify the situation.

APO A1 is the main component of HDL-C, and the two have similar clinical functions, whereas APO B is the main component of LDL-C. ${ }^{27,28}$ In addition, APO B is a major component of intermediate-density lipoprotein and very low-density lipoprotein, and hence, is recognized as a better indicator than LDL-C in cardiometabolic deases. ${ }^{29}$ Sabino et $a l^{30}$ reported that after controlling for variables, such as, age, sex, smoking, hypertension, and dyslipidemia, APO B and APO B / APO A1 ratio were independently associated with brain stroke and peripheral vascular disease.

Apolipoprotein and IOP have only been rarely investigated but were included in the present study to determine their potential uses as indicators of future IOP increases and as alternatives to HDL-C and LDL-C, which based on the discrepant results of reports are unreliable indicators.

The results of the univariate regression analysis in this study indicate that APO A1 was negatively correlated with IOP, just like HDL-C, and APO B was positively correlated with IOP, just like LDL-C and the ratio of APO B/APO A1 showed positive correlation

However, a previous univariate regression analysis on relations between apolipoproteins and IOP indicated a negative correlation with HDL-C and no correlation with APO A1. Furthermore, although increases in IOPs were observed when LDL-C and APO B levels were increased, these increases were without significance. ${ }^{25}$

The multivariate regression analysis adjusted for age, BMI, SBP, and DBP conducted in the present study showed that APO A1 was positively correlated with IOP, whereas a negative correlation was observed by univariate regression. On the other hand, APO B showed a positive correlation with IOP by univariate and multivariate regression analyses, and this correlation was stronger than those of any other lipid variable investigated. APO B/APO A1 ratio, which was investigated for the first time in this study, showed a significant positive correlation with IOP. 
Recently, a genome study related to examine IOP has been proceeded. ${ }^{31}$ Further studies may be necessary to determine whether genomic variates are correlated with other health-related indicators, such as, lipid profiles.

This study has several limitations that warrant consideration. First, it had a cross sectional design, which prevented casual relationships being determined. Second, we did not measure central corneal thickness. The association with IOP would have been more accurate if corrected IOP by central corneal thickness included in the analysis. Third, noncontact tonometry exhibits more IOP variation than Goldman applanation tonometry, even though it has been reported that non-contact tonometry is reliable within the normal IOP range. Finally, cardiovascular disease shows significant association with glaucoma or ocular hypertension have been suggested, so this is not regards as a mediator of the process affect intraocular pressure of the APO A1 and APO B, but as confounding factor. In terms of correcting the confounding factors, statistically, is adjusted for indicators associated with cardiovascular disease using multiple logistic regression. However, when stratified according to cardiovascular diseases epidemiological study design has not been performed, the effect of potential confounding factors it means that the remaining.

Nevertheless, this study is the first to examine relationships between IOP and serum apolipoprotein levels, as well as being noteworthy for analysing the relationships, adjusting for age and other risk factors, with other various lipoproteins.

In this study, APO B was found to be most strongly correlated with IOP, regardless of gender. Additional studies are required to confirm the validity of apolipoprotein level as an index for predicting IOP elevation.

\section{Summary}

What was known before

- Elevated intraocular pressure has been linked to hyperlipidemia.

What this study adds

- Apolipoprotein B showed the highest correlation with elevated intraocular pressure among all the lipid profiles.

\section{Conflict of interest}

The authors declare no conflict of interest.

\section{Acknowledgements}

This study was supported by Samsung Biomedical Research Institute grant (SMX1132271).

\section{References}

1 Sommer A. Intraocular pressure and glaucoma. Am I Ophthalmol 1989; 107(2): 186-188.

2 Le A, Mukesh BN, McCarty CA, Taylor HR. Risk factors associated with the incidence of open-angle glaucoma: the visual impairment project. Invest Ophthalmol Vis Sci 2003; 44 (9): 3783-3789.

3 Sommer A, Tielsch JM, Katz J, Quigley HA, Gottsch JD, Javitt J et al. Relationship between intraocular pressure and primary open angle glaucoma among white and black Americans. The Baltimore Eye Survey. Arch Ophthalmol 1991; 109(8): 1090-1095.

4 Leske MC, Connell AM, Wu SY, Hyman LG, Schachat AP. Risk factors for open-angle glaucoma. The Barbados Eye Study. Arch Ophthalmol 1995; 113(7): 918-924.

5 Goldberg I. Relationship between intraocular pressure and preservation of visual field in glaucoma. Surv Ophthalmol. 2003; 48(Suppl 1): S3-S7.

6 Carel RS, Korczyn AD, Rock M, Goya I. Association between ocular pressure and certain health parameters. Ophthalmology 1984; 91(4): 311-314.

7 Schulzer M, Drance SM. Intraocular pressure, systemic blood pressure, and age: a correlational study. $\mathrm{Br} J$ Ophthalmol 1987; 71(4): 245-249.

8 Kahn HA, Leibowitz HM, Ganley JP, Kini MM, Colton T, Nickerson RS et al. The Framingham Eye Study. II. Association of ophthalmic pathology with single variables previously measured in the Framingham Heart Study. Am J Epidemiol 1977; 106(1): 33-41.

9 Shiose Y. The aging effect on intraocular pressure in an apparently normal population. Arch Ophthalmol 1984; 102(6): 883-887.

10 Bonomi L, Marchini G, Marraffa M, Bernardi P, Morbio R, Varotto A. Vascular risk factors for primary open angle glaucoma. The Egna-Neumarkt Study. Ophthalmology 2000; 107(7): 1287-1293.

11 Leske MC, Podgor MJ. Intraocular pressure, cardiovascular risk variables, and visual field defects. Am J Epidemiol 1983; 118(2): 280-287.

12 Grundy SM, Pasternak R, Greenland P, Smith S Jr, Fuster V. Assessment of cardiovascular risk by use of multiplerisk-factor assessment equations: a statement for healthcare professionals from the American Heart Association and the American College of Cardiology. Circulation 1999; 100(13): 1481-1492.

13 Pasternak RC, Grundy SM, Levy D, Thompson PD. 27th Bethesda Conference: matching the intensity of risk factor management with the hazard for coronary disease events. Task Force 3.Spectrum of risk factors for coronary heart disease. J Am Coll Cardiol 1996; 27(5): 978-990.

14 Kitamura K, Yokomichi H, Yamagata Z, Tsuji M, Yoda Y, Kashiwagi K. Changes in intraocular pressure and associated systemic factors over 10 years in subjects without ocular disease at baseline. J Glaucoma 2014; 23(3): 185-189.

15 Han YS, Lee JW, Lee JS. Intraocular pressure and influencing systemic health parameters in a Korean population. Indian J Ophthalmol 2014; 62(3): 305-310.

16 Pischon T, Girman CJ, Sacks FM, Rifai N, Stampfer MJ, Rimm EB. Non-high-density lipoprotein cholesterol and apolipoprotein $\mathrm{B}$ in the prediction of coronary heart disease in men. Circulation 2005; 112(22): 3375-3383.

17 Mori K, Ando F, Nomura H, Sato Y, Shimokata H. Relationship between intraocular pressure and obesity in Japan. Int J Epidemiol 2000; 29(4): 661-666. 
18 Shiose Y. Intraocular pressure: new perspectives. Surv Ophthalmol 1990; 34(6): 413-435.

19 Lee JS, Choi YR, Lee JE, Choi HY, Lee SH, Oum BS. Relationship between intraocular pressure and systemic health parameters in the Korean population. Korean $J$ Ophthalmol 2002; 16(1): 13-19.

20 Lee GH. The association between metabolic syndrome and intraocular pressure. Korean J Health Promot 2011; 11(2): 57-63.

21 Shiose Y, Kawase Y. A new approach to stratified normal intraocular pressure in a general population. Am J Ophthalmol 1986; 101(6): 714-721.

22 Bulpitt CJ, Hodes C, Everitt MG. Intraocular pressure and systemic blood pressure in the elderly. Br J Ophthalmol. 1975; 59(12): 717-720.

23 Kim YH, Jung SW, Nam GE, Do Han K, Bok AR, Baek SJ et al. High intraocular pressure is associated with cardiometabolic risk factors in South Korean men: Korean National Health and Nutrition Examination Survey, 2008-2010. Eye (Lond) 2014; 28(6): 672-679.

24 Klein BE, Klein R, Linton KL. Intraocular pressure in an American community. The Beaver Dam Eye Study. Invest Ophthalmol Vis Sci 1992; 33(7): 2224-2228.

25 Chang YC, Lin JW, Wang LC, Chen HM, Hwang JJ, Chuang LM. Association of intraocular pressure with the metabolic syndrome and novel cardiometabolic risk factors. Eye (Lond) 2010; 24(6): 1037-1043.
26 Kim MJ, Park KH, Kim CY, Jeoung JW, Kim SH. The distribution of intraocular pressure and associated systemic factors in a Korean population: The Korea National Health and Nutrition Examination Survey. Acta Ophthalmol 2014; 92 (7): e507-e513.

27 Thompson G. Apoproteins: determinants of lipoprotein metabolism and indices of coronary risk. Br Heart J 1984; 51(6): 585-588.

28 Boffa MB, Marcovina SM, Koschinsky ML. Lipoprotein(a) as a risk factor for atherosclerosis and thrombosis: mechanistic insights from animal models. Clin Biochem 2004; 37(5): 333-343.

29 Walldius G, Jungner I. Apolipoprotein A-I versus HDL cholesterol in the prediction of risk for myocardial infarction and stroke. Curr Opin Cardiol 2007; 22(4): 359-367.

30 Sabino AP, De Oliveira Sousa M, Moreira Lima L, Dias Ribeiro D, Sant'AnaDusse LM, Das GraçasCarvalho M et al. ApoB/ApoA-I ratio in young patients with ischemic cerebral stroke or peripheral arterial disease. Transl Res 2008; 152(3): 113-118.

31 Hysi PG, Cheng CY, Springelkamp H, Macgregor S, Bailey JN, Wojciechowski R, Vitart V et al. Genome-wide analysis of multi-ancestry cohorts identifies new loci influencing intraocular pressure and susceptibility to glaucoma. Nat Genet 2014; 46(10): 1126-1130. 